The transformations of the group $G$ leave the ruled surface $S$ invariant. In fact, their geometric significance is merely to replace the two curves $C_{y}$ and $C_{z}$ upon $S$ by two other curves $C_{\eta}$ and $C_{\zeta}$ upon $S$, the point-to-point correspondence being again determined by the generators of $S$. The transformations of $G$ further involve the most general change of the independent variable. An invariant of system $(A)$ under the transformations of the group $G$ therefore has a significance for the ruled surface $S$ which is independent of the particular curves upon $S$ chosen as fundamental curves, and of the choice of the independent variable. Moreover this significance is of a projective nature, since any projective transformation of the surface $S$ gives rise to the same system of form $(A)$, and since the most general integrating ruled surface of $(A)$ is a projective transformation of any particular one.

Upon these considerations the lecturer based his theory of ruled surfaces which, together with some known results, contains a great variety of new ones. It is impossible to give an intelligible account of the details of this theory without greatly exceeding the space at our disposal. It is expounded in detail in the lecturer's recent treatise.* This brief abstract will suffice to indicate the character of the subject under investigation and the nature of the methods employed.

\title{
ON LOCI THE COORDINATES OF WHOSE POINTS ARE ABELIAN FUNCTIONS OF THREE PARAMETERS.
}

BY PROFESSOR J. I. HUTCHINSON.

(Read before the American Mathematical Society, September 3, 1906.)

A particular case of a surface the homogeneous coordinates of whose points are theta functions of three variables $u_{1}, u_{2}, u_{3}$ (connected by a relation $\vartheta=0$ ) is given by Humbert $\dagger$ and studied in considerable detail by means of this parametric rep-

* E. J. Wilczynski. Projective differential geometry of curves and ruled surfaces. Leipzig, B. G. Teubner, 1906.

$\dagger$ "Sur une surface du sixième ordre liée aux fonctions abéliennes de genre trois," Liouville, 1896, pp. 263-293. 
resentation. Such a surface may be regarded as a section of a spread $S$ (or three-dimensional locus) in space of four dimensions the coordinates $x_{0}, \cdots, x_{4}$ of whose points are five theta functions $\Theta_{i}\left(u_{1}, u_{2}, u_{3}\right)$ of the same order $m$ and characteristic (c). It is the partial, or complete intersection of $S$ by a flat space of three dimensions, a flat.

For brevity let the symbol $\omega_{k}(k=1, \ldots, 64)$ be used to refer to any one of the 64 systems of half-periods. The number of conditions that $\Theta_{i}$ vanish of order $r_{k}$ for the half-period $\omega_{k}$ is in general $\frac{1}{6} r_{k}\left(r_{k}+1\right)\left(r_{k}+2\right)$. The function then contains

$$
m^{3}-\frac{1}{6} \sum_{k=1}^{64} r_{k}\left(r_{k}+1\right)\left(r_{k}+2\right)
$$

linear constants. The cases in which this number reduces to 5 are of particular interest since all such functions are linearly expressible in terms of the $x_{i}$.

The degree $n$ of $S$ is the number of non-congruent solutions of three simultaneous equations of the form $\sum a_{i} x_{i}=0$, which is

$$
n=6 m^{3}-\sum r_{k}^{3}
$$

Combining this with the relation

we have

$$
m^{3}-\frac{1}{6} \sum r_{l k}\left(r_{k}+1\right)\left(r_{k}+2\right) \geqq 5
$$

$$
n-30 \geqq \sum\left(3 r_{t}^{2}+2 r_{k}\right) \text {. }
$$

Since the right member is positive, it follows that $n$ is not in general less than 30 , and for a given $n$ there are only a finite number of cases to be considered.

If, for example, we take the functions $x_{1}, \ldots, x_{4}$ employed by Humbert (1. c., page 270) and assume $x_{0}=\vartheta_{1} \vartheta_{2}$, we find $S$ to be of degree 36, since the coordinate functions vanish to the first order for 12 half-periods. Corresponding to these half-periods are 12 planes lying completely in $S$. The section of $S$ by the flat $x_{0}=0$ consists of these 12 planes, each counted twice, and two sextics $\vartheta_{1}=0, \vartheta_{2}=0$, the first of which is the one discussed by Humbert. The second can be transformed linearly into the first, since $\vartheta_{1}$ and $\vartheta_{2}$ permute by the addition of a suitable half-period.

If the $x_{i}$ are all even (or odd), the above relations are to be modified, since to the two different sets of values $u_{h},-u_{h}$ of the 
parameters corresponds only one point $x_{i}$. Denote by $2 s_{k}$, $2 t_{l}+1$ the order of vanishing at a half-period when that order is even or odd respectively. Then

$$
6 m^{3}-\sum_{k=1}^{\kappa} 8 s_{\kappa}^{3}-\sum_{l=\kappa+1}^{64}\left(2 t_{l}+1\right)^{3}=2 n
$$

and the number of linear constants in the $x_{i}$ is

$$
\frac{1}{2}\left(m^{3}+\mu\right)-\frac{1}{6} \sum_{k=1}^{\kappa} s_{l k}\left(s_{k}+1\right)\left(4 s_{k}-1\right)-\frac{1}{6} \sum_{l=\kappa+1}^{64} t_{l}\left(t_{l}+1\right)\left(4 t_{l}+5\right) \geqq 5
$$

in which $\kappa$ and $\mu$ have the following values:

1. $\kappa=64, \mu=8 ; m$ even, $\Theta$ even, characteristic zero ;

2. $\kappa=0, \mu=-8$; $m$ even, $\Theta$ odd, characteristic zero ;

3. $\kappa=32, \mu=0 ; m$ even, $\Theta$ odd, characteristic zero ;

4. $\kappa=36, \mu=-1 ; m$ odd, $\Theta\left\{\begin{array}{l}\text { even } \\ \text { odd }\end{array}\right\}$, characteristic $\left\{\begin{array}{c}\text { odd } \\ \text { even }\end{array}\right\}$;

5. $\kappa=28, \mu=1 ; m$ odd, $\Theta\left\{\begin{array}{l}\text { even } \\ \text { odd }\end{array}\right\}$, characteristic $\left\{\begin{array}{l}\text { even } \\ \text { odd }\end{array}\right\}$.

As in the general case, we deduce the condition

$$
n+3 \mu+2-\frac{1}{2} \kappa \leqq \sum_{k=1}^{\kappa}\left(3 s_{l}^{2}-s_{l}\right)+\sum_{l=\kappa+1}^{64}\left(3 t_{l}^{2}+2 t_{l}\right),
$$

which gives a restricted number of choices for $m, s_{k}, t$ when $n$ is given. The least value that $n$ can have appears to be 12 . This occurs in the two following cases :

I. Let $\alpha, \beta, \gamma$ denote three different systems of half-periods, and $(\alpha),(\beta),(\gamma)$ represent their characteristics. These can be taken odd without loss of generality. For, if $\left(c_{1}\right),\left(c_{2}\right),\left(c_{3}\right)$ denote any three characteristics, assume $(g)$ so that $\left(c_{1}+g\right)=(\alpha)$, $\left(c_{2}+g\right)=(\beta),\left(c_{3}+g\right)=(\gamma)$ in which the right members are odd. Then we have the three groups*

$$
\begin{aligned}
& \left(g_{1}\right)=\left(c_{2}+c_{3}\right)=(\beta)+(\gamma), \\
& \left(g_{2}\right)=\left(c_{3}+c_{1}\right)=(\gamma)+(\alpha), \\
& \left(g_{3}\right)=\left(c_{1}+c_{2}\right)=(\alpha)+(\beta) .
\end{aligned}
$$

Since any three groups either contain four characteristics $(\alpha)$,

* In the sense of Weber, Abel'sche Functionen, p. 19. 
$(\beta),(\gamma),(\delta)$ in common, or any two have six in common (none of them paired), it follows that $(\alpha),(\beta),(\gamma)$ can be chosen in a variety of ways. The sum $(\alpha+\beta+\gamma)$ is either odd, $(\delta)$, or even. In the first case the theta functions $\vartheta_{0}, \vartheta_{1}, \vartheta_{2}, \vartheta_{3}$ of the first order and characteristics $(0),\left(g_{1}\right),\left(g_{2}\right),\left(g_{3}\right)$ respectively vanish simultaneously for the half-periods $\alpha, \beta, \gamma$. There are five linearly independent theta functions with $m=2$ and characteristic (0) which vanish of order 2 for $\alpha, \beta, \gamma$. We may take for these $x_{i}=\vartheta_{i}^{2}(i=0, \ldots, 3), x_{4}=\Theta_{4}$, the last being any one linearly independent of the other four. The points $x_{i}$ lie on a spread $S$ of degree 12, which has 61 quadruple points corresponding to the half-periods other than $\alpha, \beta, \gamma$.

By equating to zero a theta function of the form $\Theta=\sum a_{i} x_{i}$ we obtain a surface of degree 12 in ordinary space. There are various types of such, according to the number and arrangement of the nodes of $S$ which lie in the given flat. There are only four cases in which such a surface degenerates, namely, when $\Theta=x_{i}(i=0, \ldots, 3)$. Then it becomes a sextic $T$ counted double. The sextic, counted singly, may be represented by $\vartheta_{i}=0$. There are 3 singular tangent planes and 25 double points, 8 in each of the singular planes, and one at their common point. By equating to zero other theta functions of the first order we obtain 24 twisted quartics of genus zero, 24 quintics, and 12 sextics lying on $T$.

II. If $(\alpha+\beta+\gamma)$ is even the groups

$$
\begin{aligned}
& \left(g_{1}\right)=(\beta)+(\gamma)=\left(\sigma_{1}^{\prime}\right)+\left(\sigma_{1}^{\prime \prime}\right)=\cdots=\left(\sigma_{5}^{\prime}\right)+\left(\sigma_{5}^{\prime \prime}\right), \\
& \left(g_{2}\right)=(\gamma)+(\alpha)=\left(\sigma_{1}^{\prime \prime}\right)+\left(\sigma_{1}\right)=\cdots=\left(\sigma_{5}^{\prime \prime}\right)+\left(\sigma_{5}\right), \\
& \left(g_{3}\right)=(\alpha)+(\beta)=\left(\sigma_{1}\right)+\left(\sigma_{1}^{\prime}\right)=\cdots=\left(\sigma_{5}\right)+\left(\sigma_{5}^{\prime}\right),
\end{aligned}
$$

in which $\left(\sigma_{i}\right),\left(\sigma_{i}^{\prime}\right),\left(\sigma_{i}^{\prime \prime}\right)$ are odd, are necessarily of the form indicated, any two having six characteristics common. Otherwise they would all have four in common, whence it would follow $(\alpha)+(\beta)=(\gamma)+(\delta),(\delta)$ being odd, which is contrary to the hypothesis.

The six theta functions with characteristics $(0),\left(\alpha+\sigma_{i}\right)$ $=\left(\beta+\sigma_{i}^{\prime}\right)=\left(\gamma+\sigma_{i}^{\prime \prime}\right)(i=1, \ldots, 5)$ vanish simultaneously for the half-periods $\alpha, \beta, \gamma^{*}$ These will be denoted by $\vartheta_{0}, \vartheta_{1}$,

* It is evident that the three characteristics $(a),(\beta),(\gamma)$ occur simultaneously in the five groups $\left(g_{i}\right)=\left(a+\sigma_{i}\right)$ and only in these. This is a more precise form of Weber's theorem - "Irgeud drei ungerade Charakteristiken, deren Summe gerade ist, kommen immer in mehreren Gruppen vor," loc. cit., p. 22. 
$\cdots, \vartheta_{5}$ respectively. Then $x_{i}=\vartheta_{i}^{2}(i=0,1, \ldots, 4)$ determine a spread $S^{\prime}$ of degree 12 having 61 quadruple points. As in the preceding example, to each of the half-periods $\alpha, \beta, \gamma$ corresponds a two-spread (a two-dimensional locus) of degree 4.

Since two functions $\vartheta_{i}$ vanish simultaneously for 12 halfperiods, three of which are $\alpha, \beta, \gamma$, it follows that the flats $a x_{i}+b x_{j}=0$ intersect $S^{\prime}$ in 9 of its nodes. If we require it to pass through a tenth not lying in either $x_{i}$ or $x_{j}$, there are 29 points to choose from, and hence we determine $29.15=435$ surfaces of degree 12, each having 10 quadruple points.

Every flat of the form $a x_{i}+b x_{j}+c x_{k}=0$ contains 3 nodes of $S^{\prime}$ and can be made to pass through two other nodes by properly choosing the constants. There are thus determined 20.45 $=900$ surfaces of degree 12 which have 5 quadruple points.

The coördinate flats $x_{i}=0$ contain doubly covered sextics. Consider, for example, the sextic $\vartheta_{0}=0$, which we denote by $T^{\prime}$. It has 25 nodes, 5 cubic curves $\vartheta_{i}=0(i=1, \ldots, 5)$ lying in singular tangent planes, 18 quartic (genus zero), 30 quintic, and 10 sextic twisted curves.

CoRnell UNIVERsity, August, 1906.

\section{ASSOCIATED CONFIGURATIONS OF THE CAYLEY-VERONESE CLASS.}

BY DR W. B. CARVER.

(Read before the American Mathematical Society, September 3, 1906.)

In this paper, $S_{n}$ will be used to denote a flat space of $n$ dimensions ; and the notation $C_{n, r}^{\nu}$ and the words "chiastic," "copoint," etc., will be used in the sense defined in the author's earlier paper $*$ on these configurations.

Given five planes in $S_{3}$, we can, in general, construct the polar point $P$ of any one of the five with respect to the 4-point determined by the other four planes. The five points so constructed, when joined by lines and planes, give a complete 5-point chi-

* "On the Cayley-Veronese class of configurations." Transactions Amer. Math. Society, vol. 6, pp. 534-545 (October, 1905). 